\title{
Qui croire ? Quels experts ? Que choisir ?
}

\author{
Who should we believe? Which authorities? What technique?
}

\section{Heresbach}

(C) Springer-Verlag France 2010

Dans le tourbillon et le déferlement d'informations, il est toujours confortable de garder quelques balises ou repères, qui dans le doute permettent de garder le cap, traditionnellement au nord. Afin de ne pas perdre ce cap, et de tracer la meilleure route, c'est-à-dire de promouvoir le meilleur soin au meilleur coût, nous avons parmi nos cartes plusieurs choix guidés par des routeurs, dans la presse grand public ou spécialisée, par des experts ou grands timoniers, et/ou par notre propre expertise, qui est un mélange de vécu et de lecture d'articles relatant les exploits ou mauvaises fortunes d'explorateurs du tube digestif contemporains ou passés.

La relativité du temps et la nécessité de colliger sur un carnet tous les détails de la vie à bord font que nous sommes tentés de puiser ou sommes spontanément inondés et abreuvés jusqu'à plus soif d'informations se prévalant d'être issues de capitaines, d'amiraux ou reflétant les aspirations de nos concitoyens : l'ensemble de ces données sont-elles crédibles ou bien doit-on personnellement consulter les cartes ou bases de données pour tracer sa route?

Deux exemples édifiants récents méritent de nourrir cette réflexion. Il y a peu de temps, un expert américain en «polypologie » et vidéocoloscopie avait comparé la coloscopie courte, ou rectosigmoïdoscopie (RSS), pour le dépistage du cancer colorectal (CCR), à la mammographie d'un seul sein dans un pays qui pourtant nous avait montré la voie de la sagesse en prônant le dépistage du CCR par n'importe quel moyen, à condition que le patient en assume les avantages et inconvénients! Cet expert se voit contredit, par l'evidence based medicine [1] qui publie les résultats attendus depuis 1999, date de la fin du dépistage en population par RSS. Dans cette série comparant 112939 contrôles à 57099 sujets dépistés par RSS, la mortalité spécifique était signifi-

D. Heresbach $(\bowtie)$

Rédacteur en chef Acta Endoscopica,

Président de la commission recommandation de la SFED,

Service des maladies de l'appareil digestif,

CHU de Pontchaillou, 2, rue Henri-Le-Guilloux,

F-35033 Rennes cedex 09, France

e-mail : denis.heresbach@chu-rennes.fr cativement diminuée de $31 \%$, et la mortalité globale marginalement significative $(p<0,051)$ de $3 \%$. Bien que la RSS ait une mauvaise réputation, acceptabilité ou faisabilité, les bases qui ont promu le dépistage par recherche de saignement occulte dans les selles (RSOS) n'ont montré une diminution de la mortalité spécifique par CCR que de $13 \%$ sans modifier la mortalité globale, et la RSOS dite immunologique n'a pas fait plus que la coloscopie totale ou virtuelle la démonstration de son impact en population sur la mortalité par CCR. Alors, au nom de l'efficacité, en particulier budgétaire, allons-nous suivre ce qui est écrit et publié dans les revues de qualité ou suivre l'avis d'experts influents?

L'autre voie est d'attendre que la presse, ni spécialisée, ni grand public s'empare du sujet, mais quelle est leur capacité à relayer une information spécialisée de façon fiable : il y a peu de temps, un magazine censé éclairer le consommateur a fait réagir quelques-uns de nos collègues à propos de la polypectomie d'un à trois polypes de moins de $1 \mathrm{~cm}$ et surtout de la durée de séjours qui y était associée et de l'accessibilité aux données PMSI [2]. Cet article dénonçant certains abus souligne cependant le respect des honoraires pour cet examen et que la différence de tarification aux patients (ou à leur mutuelle) est largement consécutive aux frais hôteliers. Il est dommage que cet article soit paru avant deux articles parus dans la presse spécialisée : celui [3] qui a démontré que l'incidence des CCR d'intervalle était corrélée de façon indépendante aux taux de détection des adénomes pendant la vidéocoloscopie dans une large série menée en population, témoignant ainsi de l'efficacité de cet examen lorsqu'il est réalisé selon les règles de l'art, et par ailleurs celui qui a démontré en population que la coloscopie courte diminue de façon significative de 23 et $31 \%$ l'incidence et la mortalité par CCR (en intention de dépister) 11 ans après un dépistage par RSS souple unique entre 55 et 64 ans et de façon significative la mortalité globale.

Nous disposons donc aujourd'hui de deux pierres supplémentaires (à prix modéré et respecté) qui permettent de démontrer le bien-fondé d'une prévention et non d'un simple dépistage du CCR, même si le retentissement sur la mortalité en population n'est démontré (pour l'instant) que pour la 
coloscopie dite courte : pourquoi en serait-il différent pour la coloscopie longue avec un taux de détection des adénomes adéquats?

L'objectivité des experts et de la presse doit donc nous inciter à choisir notre route et à ne pas enfermer notre exercice dans la lecture d'un roadbook simplifié ou simpliste. Une formation trop conventionnelle n'est pas compatible avec la créativité scientifique, qui demande de l'imagination et du non-conformisme pour sortir des sentiers battus. Mais pour en sortir, encore faut-il les connaître et les accepter !

\section{Références}

1. Atkin WS, Edwards R, Kralj-Hans I, Wooldrage K, Hart AR, Northover JM, et al. Once-only flexible sigmoidoscopy screening in prevention of colorectal cancer: a multicentre randomised controlled trial. Lancet 2010;375:1624-33.

2. Que choisir. 2010, pp. 16-24.

3. Kaminski MF, Regula J, Kraszewska E, Polkowski M, Wojciechowska U, Didkowska J, et al. Quality indicators for colonoscopy and the risk of interval cancer. N Engl J Med 2010; 362:1795-803. 